\title{
miR-762 can negatively regulate menin in ovarian cancer
}

This article was published in the following Dove Press journal:

OncoTargets and Therapy

12 April 2017

Number of times this article has been viewed

\section{Rui Hou' \\ Zhuo Yang' \\ ShiZhuo Wang' \\ DaMing Chu' \\ Qifang Liu' \\ Jia Liu' \\ Luo Jiang ${ }^{2}$}

'Department of Obstetrics and Gynecology, ${ }^{2}$ Department of Ultrasound, Shengjing Hospital

Affiliated to China Medical University,

Shenyang, People's Republic of China
Correspondence: Luo Jiang

Department of Ultrasound, Shengjing Hospital Affiliated to China Medical

University, 36 Sanhao Street,

Heping District, Shenyang I 10004 ,

People's Republic of China

Tel/fax +86 249661533 III

Email jiangluo2016@126.com
Abstract: Ovarian cancer accounts for the major part of the mortality attributable to female reproductive system malignant tumors worldwide. Recently, the incidence of ovarian cancer has been increasing annually, and there remains a lack of suitable treatment methods that can significantly improve the 5 -year survival rates of patients. Therefore, it is necessary to identify more effective treatments for ovarian cancer. It is established that microRNAs (miRNAs) have important roles in the diagnosis and treatment of ovarian cancer and a specific miRNA, miR-762, can promote the development of a variety of tumors. Menin is encoded by MEN1, a tumor suppressor gene, that is usually downregulated in ovarian cancer. In this study, we evaluated the expression levels of miR-762 and menin in ovarian cancer tissues and demonstrated that they were correlated. In addition, we found that miR-762 can downregulate the expression of menin through a binding site in its 3 '-UTR and consequently upregulate the Wnt cell signaling pathway to promote the development of ovarian cancer. These results indicate that miR-762 is a promising potential target for the treatment of ovarian cancer.

Keywords: miR-762, menin, ovarian cancer

\section{Introduction}

Ovarian cancer is the most lethal gynecological tumor worldwide, and its incidence remains high. Statistical data indicate that, in the USA alone, ovarian cancer accounted for an estimated 22,240 new cases and 14,030 deaths in 2013. ${ }^{1}$ The early clinical course of ovarian cancer is asymptomatic, and it is difficult to distinguish the benign and malignant histological types of the disease. ${ }^{2}$ In $\sim 30 \%$ of patients with ovarian cancer, the tumor is confined to the ovary at diagnosis and can be removed by laparotomy; however, in the majority of cases, the malignancy spreads to the uterus, adnexa, or pelvic organs, and hence, there remain serious issues surrounding the timely diagnosis and treatment of ovarian cancer. ${ }^{3}$ Over the years, there has been a great deal of research into the pathology, clinical development, and treatment of ovarian cancer; however, the overall cure rate remains at only $30 \%{ }^{4}$ Additional studies, taking advantage of contemporary technologies to identify molecular alterations in ovarian cancer, are therefore urgently required to facilitate the development of strategies for earlier diagnosis and more effective therapy.

A group of noncoding RNA molecules, microRNAs (miRNAs), can repress multiple target genes through the endogenous cellular mechanism, RNA interference. Dysregulation of miRNAs has been reported in a variety of human cancers, and their expression is closely associated with patient prognosis..$^{5-7}$ Accumulating evidence demonstrates that the biological functions of miRNAs are realized through the regulation of target cell signaling networks. In ovarian cancer, numerous miRNAs have been suggested to have functional 
diagnostic, prognostic, and therapeutic implications. Iorio et $\mathrm{al}^{8}$ reported that miR-141, miR-200a, miR-200b, and miR-200c were upregulated and miR-199a, miR-140, miR-145, and miR-125b-1 were downregulated in ovarian cancer tissues/cell lines. Moreover, Calura et $\mathrm{al}^{9}$ identified robust miRNA markers for clear cell and mucinous histotypes through the analysis of miRNA profiles characteristic of stage 1 epithelial ovarian cancers. Furthermore, the miRNAs, miR-146a and miR-150, are significantly overexpressed in omental metastases of ovarian cancer and may regulate enhanced spheroid formation and cisplatin resistance. ${ }^{10}$ Overall, these reports indicate that miRNAs regulate multiple targets and affect various biological functions in ovarian cancer cells.

miR-762 is upregulated in radiation-induced tumors in mice and may affect pathways involved in apoptosis in this context. ${ }^{11}$ Expression of miR-762 is high in both breast cancer cell lines and clinical specimens, and its overexpression increases breast cancer cell proliferation and invasion. miR-762 may function by targeting interferon regulatory transcription factor 7 (IRF7) in human breast cancer. ${ }^{12}$ Previous research also demonstrated an association between miR-762 expression and oral carcinogenesis, ${ }^{13}$ however, there are no reports detailing the expression and function of miR-762 in ovarian cancer.

Menin is a protein encoded by the gene, multiple endocrine neoplasia type 1 (MEN1), mutations of which are the predominant cause of the hereditary disorder, MEN1 syndrome. Menin has various biological roles that affect embryo growth and development, and it is closely associated with tumor occurrence and growth, with a reported function as a tumor suppressor. ${ }^{14}$

In this study, we investigated the expression of miR-762 and menin in ovarian cancer and evaluated the relationship between them. The results indicate that miR-762 is associated with ovarian cancer and can promote the growth and metastasis of ovarian cancer cells through the downregulation of menin expression.

\section{Materials and methods Tissue samples and cell lines}

Paired tumor and adjacent normal tissue samples $(n=60)$ were obtained from patients with ovarian cancer in the Shengjing Hospital Affiliated to China Medical University. The study protocols were approved by the ethics committee of Shengjing Hospital Affiliated to China Medical University. All cases were diagnosed with ovarian cancer and treated between May 2009 and June 2010 at the Shengjing Hospital Affiliated to China Medical University.

The human ovarian adenocarcinoma cell lines, SKOV3 and CAOV (purchased from Shanghai Maisha
Biotechnology, Shanghai, People's Republic of China), were cultured in RPMI-1640 medium supplemented with $10 \%$ fetal bovine serum (GE Healthcare Bio-Sciences Corp., Piscataway, NJ, USA ), under $5 \% \mathrm{CO}_{2}$ at $37^{\circ} \mathrm{C}$.

\section{MTT cell viability assays}

Cells were seeded at a density of $1 \times 10^{3} /$ well in 96-well plates and transfected with miR-762 mimic, GGGGCUGGGGCC GGGGCCGAGC (miR10010301-1-5), negative control, UCACAACCUCCUAGAAAGAGUAGA (miR01201-1-5), or miR-762 inhibitor, CCCCGACCCCGGCCCCGGCUCG (miR20010313-1-5), and $12 \mathrm{~h}$ later, cell proliferation was evaluated using an MTT assay. Optical densities were measured at $490 \mathrm{~nm}$ using a microplate reader (Bio-Rad Laboratories Inc., Hercules, CA, USA).

\section{Metastasis assays}

Modified Boyden chambers containing polycarbonate nucleopore membranes (24-well plates, 8 - $\mu \mathrm{m}$ pore size; Corning) were used to perform metastasis assays according to the manufacturer's specifications. Twenty-four hours after transfection with miR-762 mimic, miR-762 inhibitor, or control sequences, cells were seeded in transwell chambers in serum-free media with Matrigel membranes covered or uncovered with medium containing $0.1 \%$ bovine serum albumin, while medium containing 30\% fetal bovine serum was placed in the lower chambers. After $24 \mathrm{~h}$, cells underneath the membrane were stained with $0.1 \%$ crystal violet and counted under a microscope.

\section{Real-time PCR}

Total RNA from tissue samples or cells was extracted using TRIzol (Thermo Fisher Scientific, Waltham, MA, USA) and standard protocols. Real-time PCR was performed using the real-time PCR Universal Reagent on an MX3000P instrument, following the manufacturer's instructions. Primer sequences are presented in Table 1 , and all reactions were carried out as described previously. ${ }^{15}$

\section{Western blot analyses}

Cells and tissues were lysed in lysis buffer, supplemented with proteinase and phosphatase inhibitors (Sigma-Aldrich, St Louis, MO, USA). To determine the expression levels of specific proteins, $30 \mu \mathrm{g}$ aliquots of total proteins from each sample were subjected to electrophoresis on $10 \%$ sodium dodecyl sulfate (SDS)-polyacrylamide gels and transferred onto nitrocellulose membranes (Millipore Corporation, Billerica, MA, USA). Target proteins were probed with specific antibodies as follows: menin (sc-374371), $\beta$-catenin 
Table I Primer sequences for the detection of RNA expression

\begin{tabular}{lll}
\hline Name & Forward primer $\left(\mathbf{5}^{\prime} \rightarrow \mathbf{3}^{\prime}\right)$ & Reverse primer $\left(\mathbf{5}^{\prime} \rightarrow \mathbf{3}^{\prime}\right)$ \\
\hline miR-762 & ACACGGGGCUGGGGCCGGGGCCGAGCGCCTC & CTCAGGGGCUGGGGCCGGGGCCGAGCCAGA \\
U6 & CTCGCTTCGGCAGCACA & AACGCTTCACGAATTTGCGT \\
Menin & GCACACAGACAACCTGATCTTTT & TCGGGAACGTTGGTAGGGAT \\
BCL-2 DI & CCGAGGAGCTGCTGCAAATGGAG & GAAATCGTGCGGGGTCATTGCG \\
BAX & GGTGAACTGGGGGAGGATTG & GGCAGGCATGTTGACTTCAC \\
MMP7 & AGCTGAGCGAGTGTCTCAAG & GTCCAATGTCCAGCCCATGA \\
GAPDH & ACAATTGTCTCTGGACGGCA & TAGTCCTGAGCCTGTTCCCA \\
\hline
\end{tabular}

(sc-53483), cyclin D1 (sc-4074), BAX (sc-4239), BCL-2 (sc-56015), MMP7 (sc-26680), and GAPDH (sc-365062) (Santa Cruz Biotechnology Inc., Dallas, TX, USA). Relative expression levels were determined using ImageJ software.

\section{Dual luciferase reporter assay}

Dual luciferase activity assays were performed as previously described. ${ }^{16}$ The menin $3^{\prime}$-UTR was PCR amplified and cloned into the pMIR-REPORTTM vector (Ambion, Shanghai, People's Republic of China), using the primers menin-wt, F: 5'-GGATTGCCGGAGTTCGAAGA-3' and R: 5'-CGACGCGGTAACACCATTTG-3', and menin-del, F: 5'-CACGGTCCTAAGCCAGTAGC-3' and R: 5'-CGACAAACAGGGACGCAATG-3'. SKOV3 cells were cotransfected with $500 \mathrm{ng}$ of wild type or deleted menin 3'-UTR reporter vector and $10 \mathrm{ng}$ of pMIR-REPORTTM- $\beta$ gal control plasmid, along with miR-762 mimic or miR-762 antisense (as inhibitor). Luciferase activity was determined $36 \mathrm{~h}$ after transfection using a Dual Luciferase ${ }^{\circledR}$ Reporter Assay System (Promega Corporation, Fitchburg, WI, USA).

\section{Statistical analyses}

Correlation analysis using a log-rank test was used to evaluate the relationships among the expression levels of potential prognostic factors. Differences were analyzed by analysis of variance (ANOVA) tests. All data were analyzed using SPSS17.0 (SPSS, Chicago, IL, USA). Statistical significance was defined as $P<0.05$. All experiments were performed in triplicate.

\section{Ethics statement}

All methods were carried out in accordance with relevant guidelines and regulations. All the patients were asked to check the study protocol and understand its significance before providing signed informed consent. The study protocol was approved by the Ethics Committee of the Shengjing Hospital and Human Clinical Trial Committee, with reference to the Chinese Community Guidelines. Written informed consent was obtained from all patients.

\section{Results}

\section{The relationship between miR-762 and} menin in ovarian cancer

Examination of the expression of miR-762 in ovarian cancer and adjacent tissue samples from 60 patients demonstrated that the expression of miR-762 was upregulated in tumor relative to adjacent nontumor tissue (Figure 1A). Next, we analyzed the association between miR-762 expression and survival in ovarian cancer. The results of Kaplan-Meier analysis indicated that cases with high levels of miR-762 expression had significantly worse overall survival than those with low levels of expression (Figure 1B). We then investigated the relationship between menin expression and ovarian cancer by determining expression levels of menin in ovarian cancer and adjacent tissues by Western blot and real-time PCR analyses (Figure 1C and D). The results demonstrated lower expression of menin in the ovarian cancer tissues than in the adjacent normal tissues.

To explore the relationship between miR-762 and MEN1 expressions in ovarian cancer, we analyzed the correlation of their real-time PCR expression results and found that they exhibited a negative correlation (Figure 1E). Next, using the miRDB software, we identified an miR-762-binding site within the menin $3^{\prime}$-UTR sequence (Figure 1F). Luciferase reporter assays demonstrated that miR-762 can indeed directly reduce the expression of menin at the transcriptional level (Figure 1G), since cells cotransfected with a luciferase reporter construct containing the wild-type menin $3^{\prime}$-UTR and miR-762 generated reduced luciferase activity compared to controls; however, no significant variation in luciferase levels was observed in cells cotransfected with the menindel luciferase reporter or miR-762 antisense (miR-762 inhibitor).

\section{miR-762 can inhibit the proliferation of ovarian cancer cells by targeting menin}

An MTT assay was used to examine the effect of miR-762 on the proliferation of ovarian cancer cells. Transfection with miR-762 mimic resulted in a significantly enhanced 
A

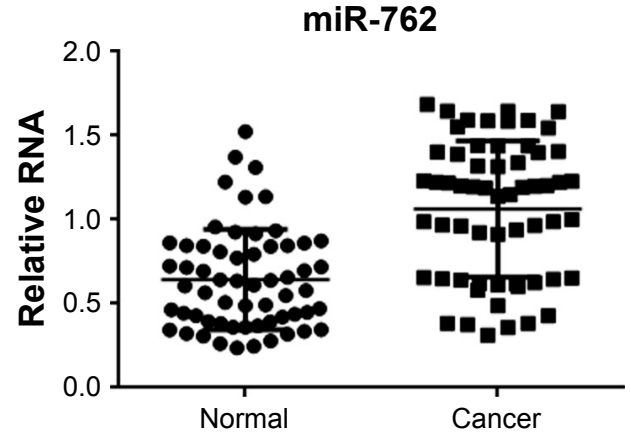

C

$\begin{array}{llllllllllllllll}\mathbf{N} & C & N & C & N & C & N & C & N & C & N & C & N & C\end{array}$

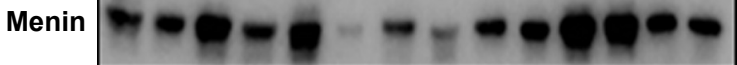

GAPDH

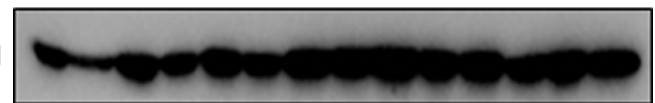

D

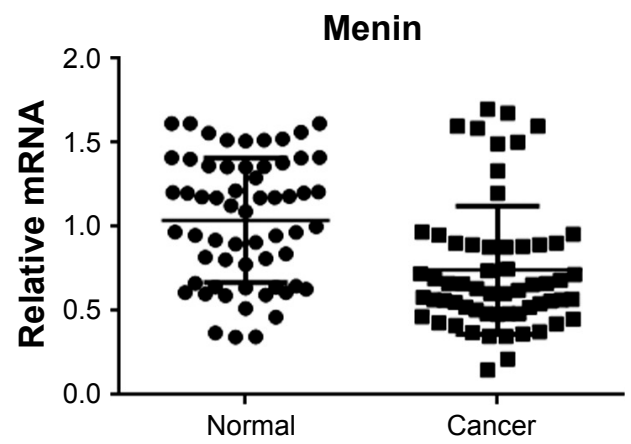

$\mathbf{F}$

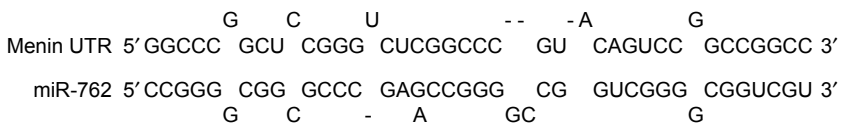

B
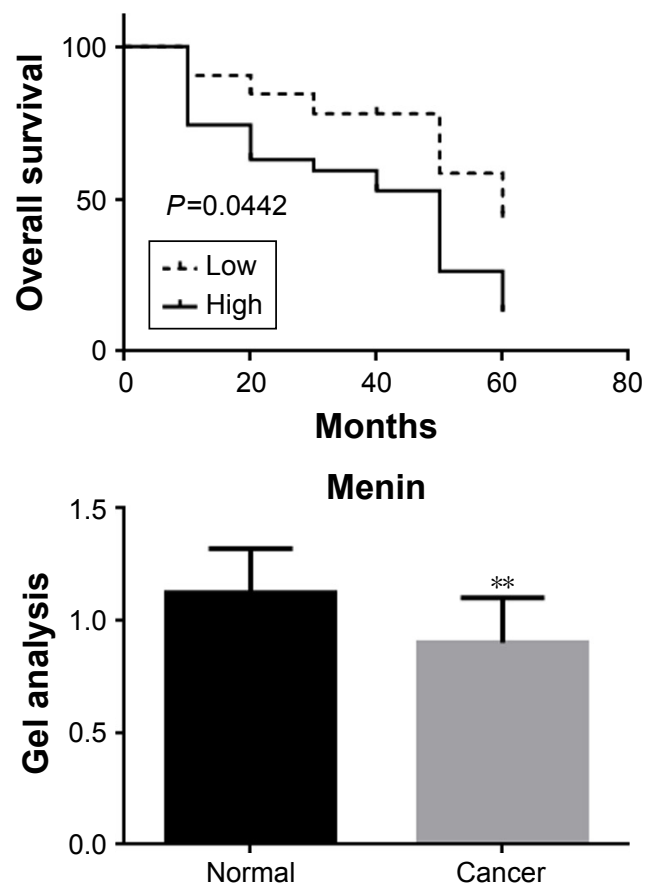

E

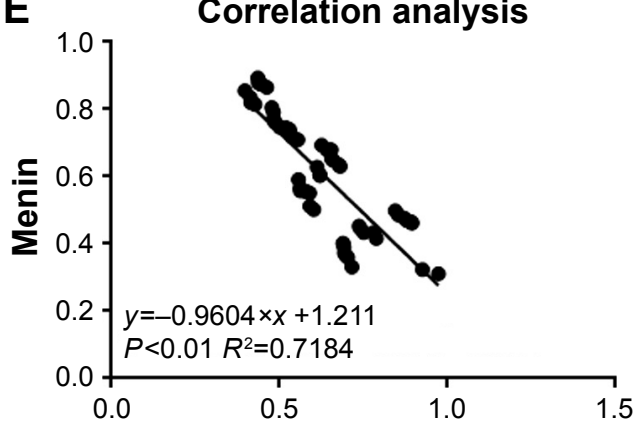

G

miR-762

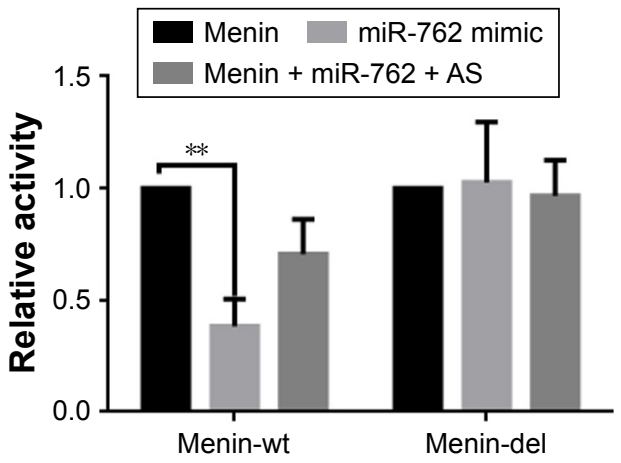

Figure I The relationship between miR-762 and menin in ovarian cancer.

Notes: (A) The levels of miR-762 were detected by real-time PCR in 60 samples of ovarian cancer and adjacent tissues. There was higher expression of miR-762 in ovarian cancer tissues. Data are presented as mean \pm SEM. (B) The relationship between miR-762 expression levels and patient survival. The survival time of patients with high expression of miR-762 was shorter than that of patients with low expression. (C) Menin protein levels in ovarian cancer (C) and adjacent tissues ( $N$ ) were detected by Western blotting. The expression level of menin was lower in the ovarian cancer tissues than in the control tissues. Quantitative data (right) are presented as mean \pm SEM; $* * P<0.01$ tumor tissue group vs adjacent control tissue group. (D) mRNA expression of MENI in 60 samples of ovarian cancer and adjacent tissues was detected by real-time PCR. The expression of menin was lower in ovarian cancer tissues than in the control tissues. Data are presented as mean $\pm \mathrm{SEM}$. $* * P<0.0 \mathrm{I}$ for the comparison of tumor tissue group vs adjacent control tissue group. (E) Correlation between the expression levels of miR-762 and menin. The expression of miR-762 was negatively correlated with that of menin. (F) Prediction that miR-762 can specifically bind to the menin 3'-UTR using miRDB. (G) The interaction between miR-762 and the menin $3^{\prime}$-UTR was evaluated using luciferase reporter assays. Assays were performed by the cotransfection of miR-762 or miR-762 antisense and menin-wt or menin-del. Data are presented as mean \pm SEM. $* * P<0.01$ vs TK-menin group.

Abbreviations: SEM, standard error of mean; PCR, polymerase chain reaction; GAPDH, glyceraldehyde-3-phosphate dehydrogenase; UTR, untranslated regions. 
proliferation of SKOV3 and CAOV cells (Figure 2A). In contrast, transfection with miR-762 inhibitor led to suppression of ovarian cancer cell proliferation (Figure 2B). Cyclin D1 is a protein in the $\mathrm{Wnt} / \beta$-catenin signaling pathway involved in cell proliferation. Therefore, we analyzed the levels of Cyclin D1, $\beta$-catenin, and menin protein and mRNA after transfection of SKOV3 cells with miR-762 mimic or inhibitor (Figure 2C-F). When miR-762

\section{A}
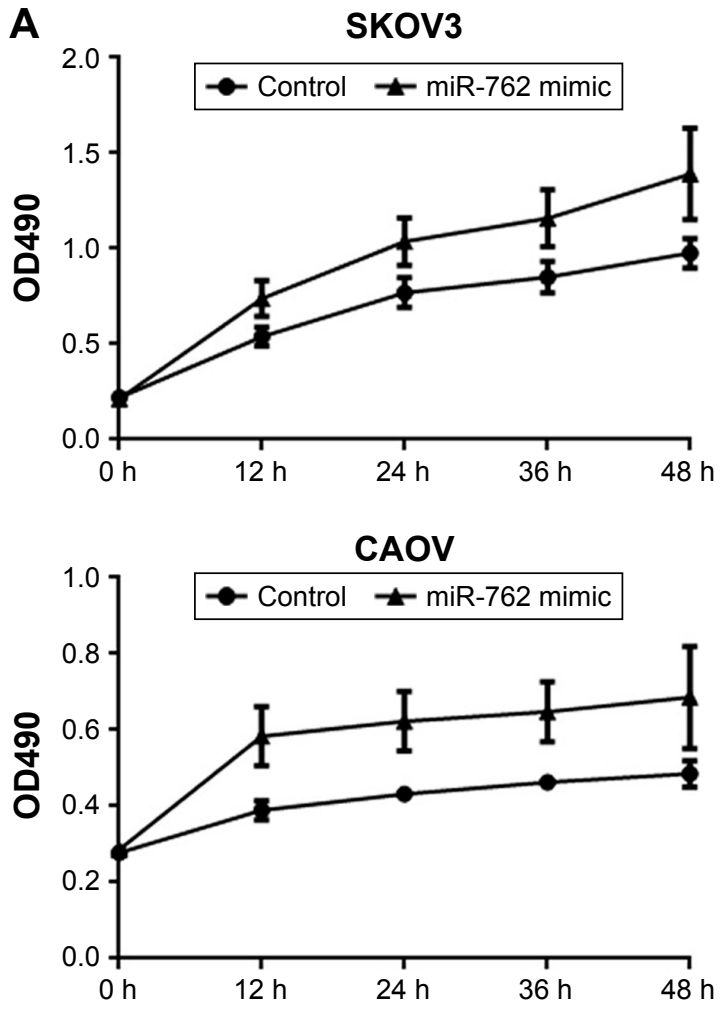

B

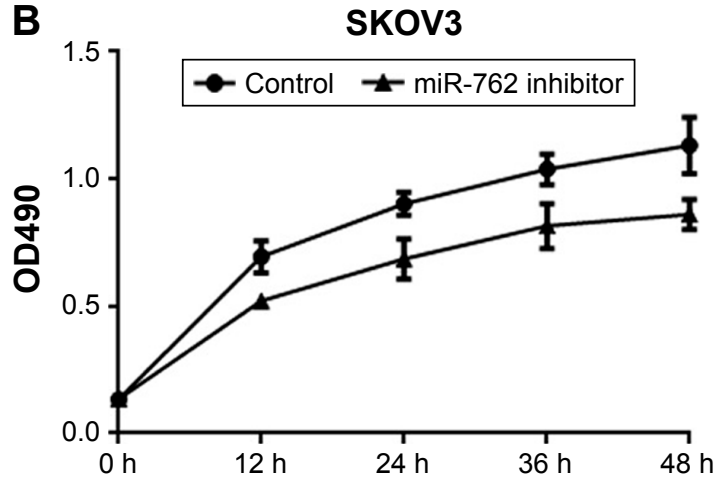

CAOV

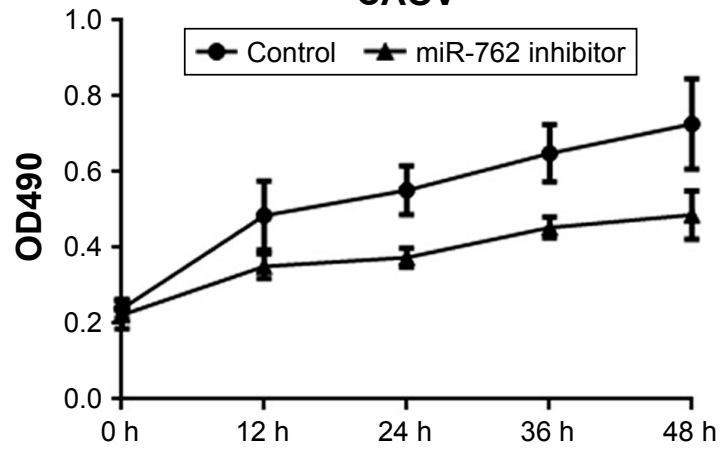

SKOV3
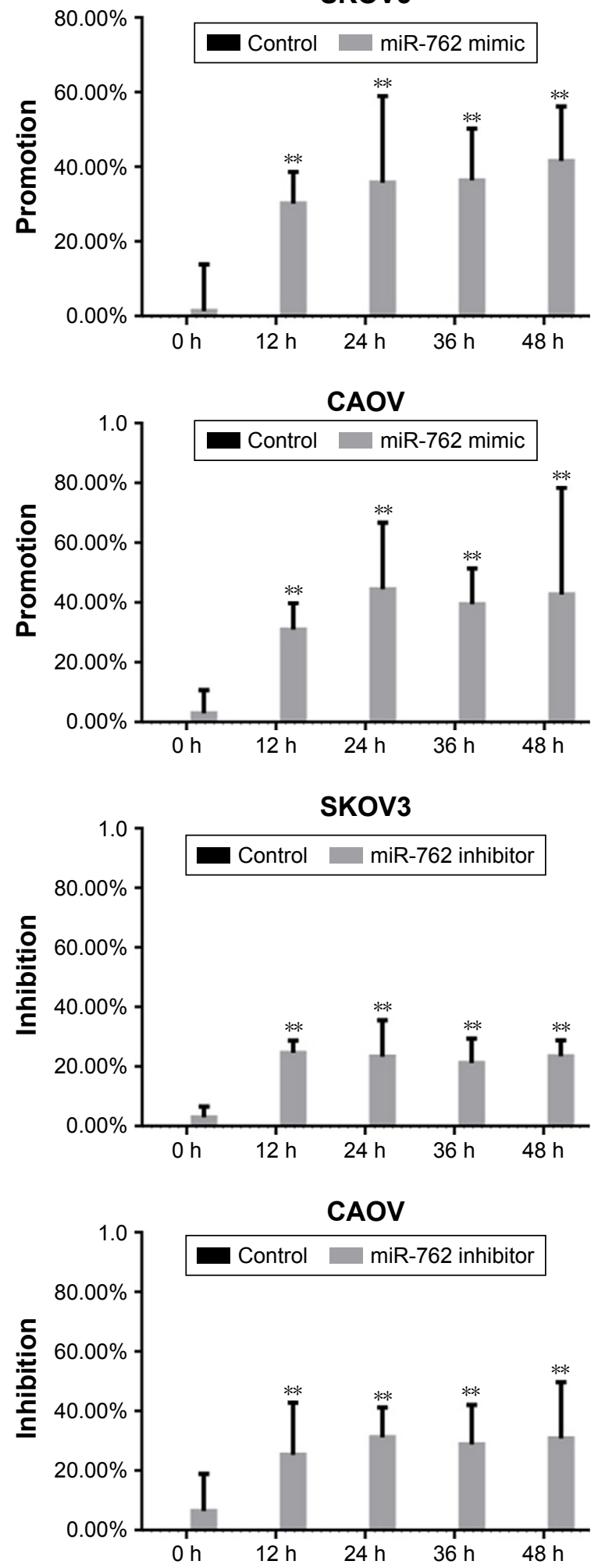

Figure 2 (Continued) 

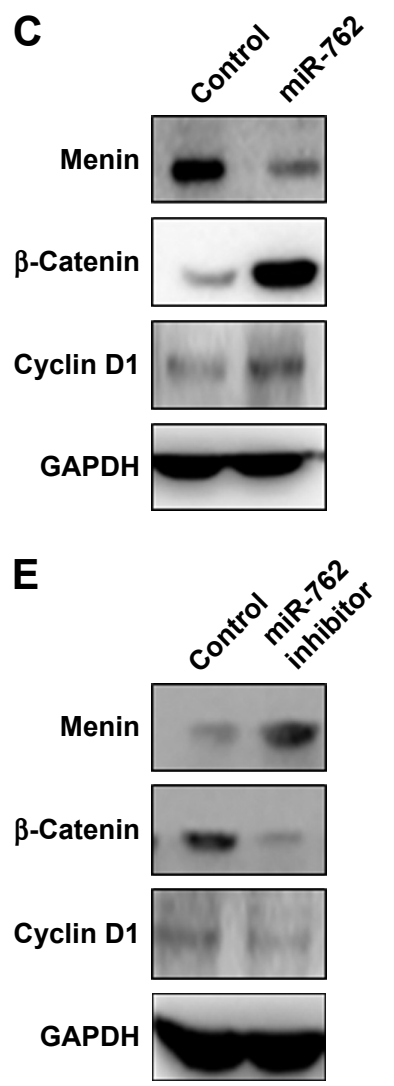

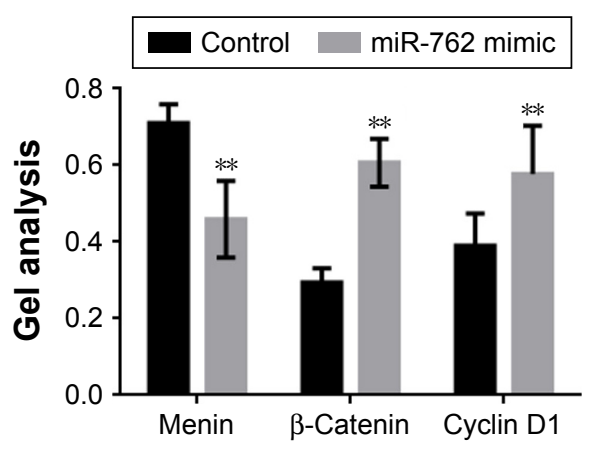

D

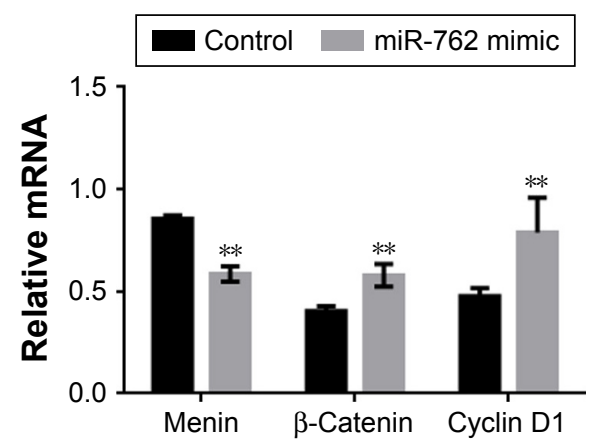

$\mathbf{F}$
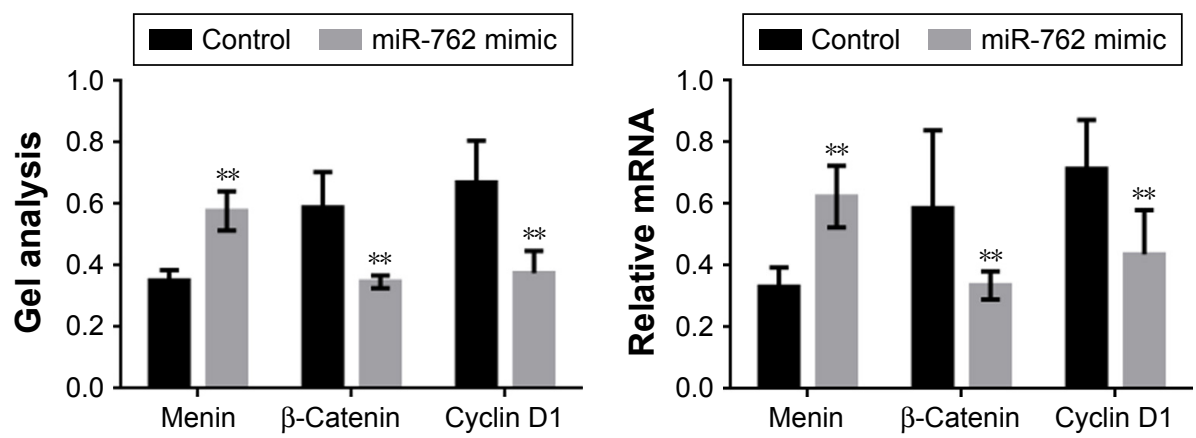

Figure 2 miR-762 can promote the proliferation of ovarian cancer cells by targeting menin.

Notes: (A) After transfecting with miR-762 mimic, the proliferation rates of SKOV3 and CAOV cells were determined using MTT assays. Data are presented as mean \pm SEM. (B) After transfection with miR-762 inhibitor, the levels of proliferation of SKOV3 and CAOV cells were determined using MTT assays. Data are presented as mean \pm SEM. (C and D) After transfecting SKOV3 cells with miR-762 mimic, the protein and mRNA levels of menin, $\beta$-catenin, and cyclin DI were detected by Western blotting and realtime PCR, respectively. Data are presented as mean \pm SEM. $* * P<0.01$. (E and $\mathbf{F}$ ) After transfection of SKOV 3 cells with miR-762 inhibitor, the protein and mRNA levels of menin, $\beta$-catenin, and cyclin $D I$ were detected by Western blotting and real-time $P C R$. Data are presented as mean $\pm S E M$. $* * P<0.01$.

Abbreviation: MTT, 3-(4,5-Dimethylthiazol-2-yl)-2,5-diphenyltetrazolium bromide.

was overexpressed in SKOV3 cells, menin was downregulated, while the expression of $\beta$-catenin and cyclin D1 was promoted. Conversely, after the inhibition of miR-762, the expression of menin was increased, while $\beta$-catenin and cyclin D1 expressions were inhibited. These results suggest that miR-762 can affect the proliferation of ovarian cancer cells through regulation of the expression of menin and the Wnt/ $\beta$-catenin signaling pathway.

\section{miR-762 inhibits the apoptosis of ovarian cancer cells by suppressing the Wnt/ $\beta$-catenin pathway}

Next, we examined the expression of the key regulators of apoptosis, BAX and BCL-2, by Western blotting and realtime PCR in the context of overexpression or downregulation of miR-762 (Figure 3A-D). The results demonstrated that miR-762 can inhibit the expression of menin and BAX; however, it significantly increased the expression of $\beta$-catenin and BCL-2. Overall, these results indicate that miR-762 can negatively regulate menin and, consequently, promote the proliferation and inhibit the apoptosis of SKOV3 cells through regulation of the $\mathrm{Wnt} / \beta$-catenin signaling pathway.

\section{miR-762 promotes the metastasis of ovarian cancer cells by suppressing menin}

Transwell assays (with or without Matrigel) were used to determine whether miR-762 can affect phenotypes associated with metastasis (migration and invasion) in ovarian cancer cells (Figure 4A-D). Migration and invasion of SKOV3 and CAOV cells were significantly promoted when miR-762 was overexpressed; however, when miR-762 expression was knocked down, their migration and invasion were inhibited. In addition, Western blotting and real-time PCR were used to determine the protein and mRNA levels of menin, $\beta$-catenin, and MMP7 in SKOV3 cells (Figure 4E-H). Menin was significantly downregulated in response to miR-762 expression, while $\beta$-catenin and MMP7 were significantly upregulated. These experiments confirm that miR-762 can 

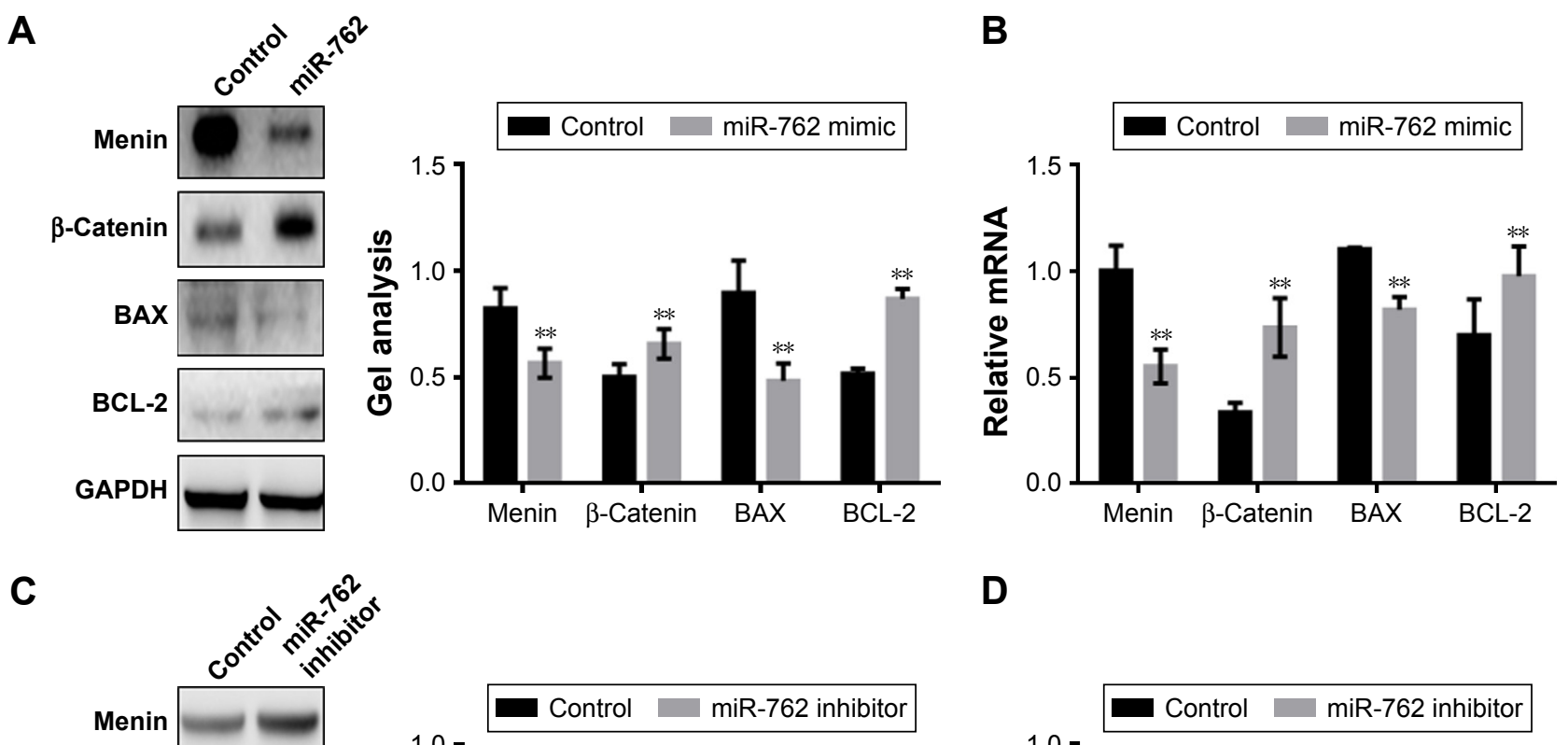

D

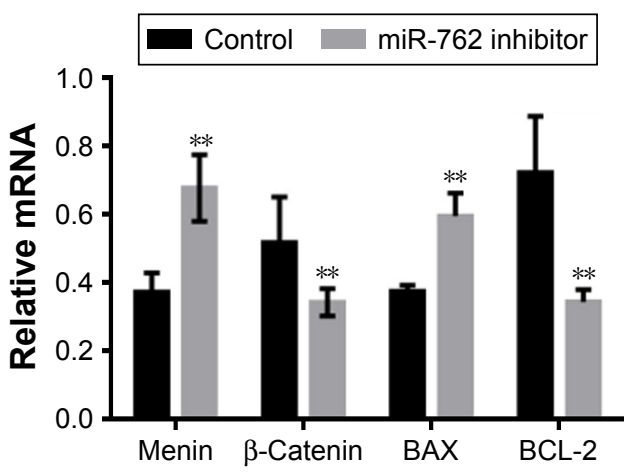

Figure 3 miR-762 inhibits the apoptosis of ovarian cancer cells by suppressing the $\mathrm{Wnt} / \mathrm{\beta}$-catenin pathway.

Notes: (A and B) After transfection of SKOV3 cells with miR-762 mimic, the protein and mRNA levels of menin, $\beta$-catenin, BAX, and BCL-2 were determined by Western blotting and real-time PCR. Data are presented as mean \pm SEM. $* * P<0.01$. (C and D) After transfection of SKOV3 cells with miR-762 inhibitor, the protein and mRNA levels of menin, $\beta$-catenin, BAX, and BCL-2 were detected by Western blotting and real-time PCR. Data are presented as mean $\pm S E M$. $* * P<0.01$.

Abbreviation: SEM, standard error of mean.

promote phenotypes associated with metastasis in ovarian cancer cells through the regulation of menin.

\section{Discussion}

Experimental studies indicate that menin can inhibit the expression of SOX4 and, consequently, regulate early embryonic and nervous system development. SOX4 may function in the apoptosis pathway, leading to cell death, as well as tumorigenesis, and may also mediate the downstream effects of parathyroid hormone (PTH) and PTH-related protein (PTHrP) in bone development. Menin also inhibits the transcriptional expression of $P A X 2$ to regulate Wilms' tumor, ${ }^{17}$ induces cellular DNA repair, and promotes apoptosis. ${ }^{18-21}$ Furthermore, menin can interact with JunD to block cell proliferation, interact with Smad to affected the transforming growth factor signal transduction pathway, and regulate the cell cycle through its influence on cyclin-dependent kinases. Moreover, there is evidence that menin may be involved in regulation of the Wnt and MAPK signaling pathways, ${ }^{18-21}$ which are closely related to the occurrence and development of tumors. Hence, menin is a potential therapeutic target in various cancers.

Previous studies have reported abnormal miRNA expression in ovarian cancer, and miR-762 overexpression has been reported in breast cancer and thymic lymphoma, where it could promote cancer cell growth and metastasis. ${ }^{12}$

Although miR-762 is overexpressed in numerous cancers, there were no previous reports of investigations of miR-762 in ovarian cancer; therefore, we evaluated miR-762 in this context. In our study, we confirmed by real-time PCR that the expression of miR-762 in ovarian cancer was almost twice that in the adjacent tissues. There was also an association between miR-762 expression and the survival time of patients with ovarian cancer, where patients with high expression of miR-762 exhibited shorter survival times than those with low expression of miR-762. As a tumor suppressor gene, menin has been shown to inhibit the occurrence and development of a variety of tumors. ${ }^{22}$ When menin is overexpressed, it can 
reduce $\beta$-catenin nuclear accumulation and its transcriptional activity, ${ }^{23}$ and there is ample evidence that it can regulate cell growth and metastasis through the suppression of $\mathrm{Wnt} / \beta$-catenin signaling. ${ }^{22,23} \mathrm{We}$ wished to determine whether
miR-762 could affect the $\beta$-catenin pathway through targeting of menin. The expression of menin in ovarian cancer tissues, at both the protein and mRNA levels, was lower than that in samples from adjacent control tissue. Meanwhile, statistical
A
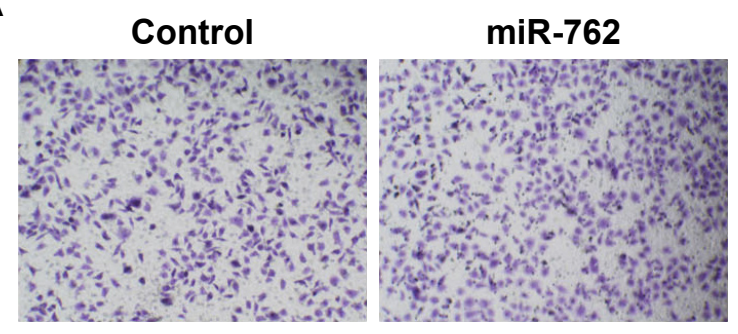

Control

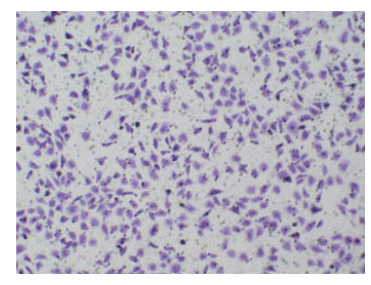

B

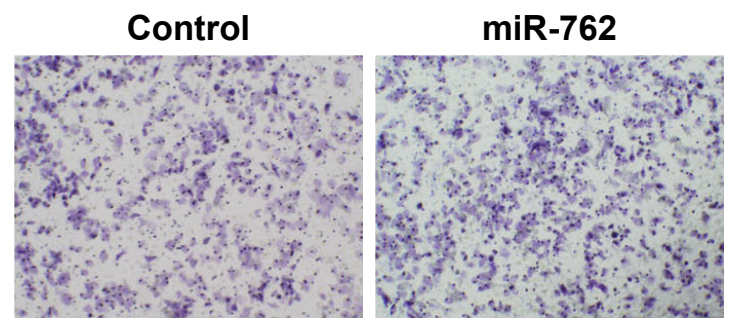

\section{Control}

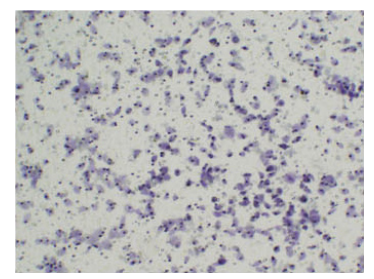

C

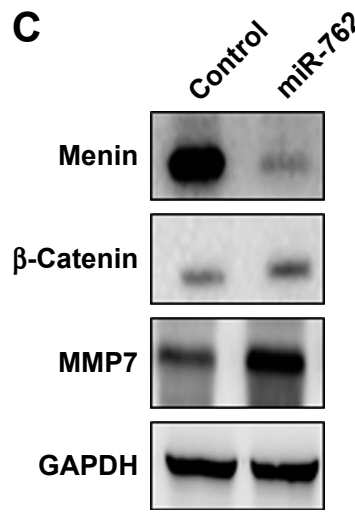

miR-762

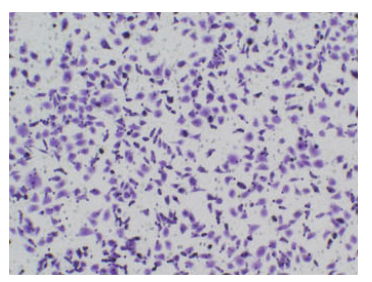

miR-762

miR-762

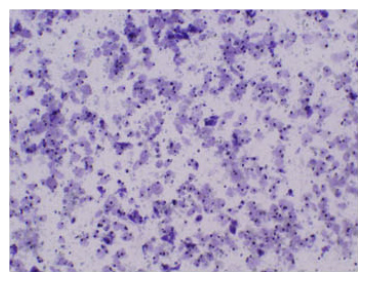

Control miR-762 mimic

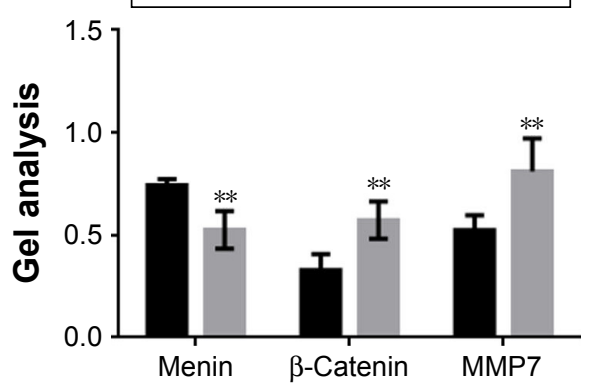

SKOV3 migration

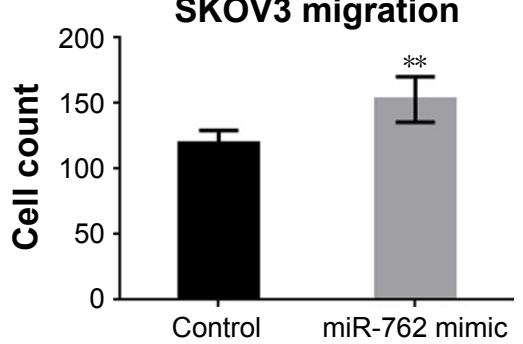

SKOV3 invasion
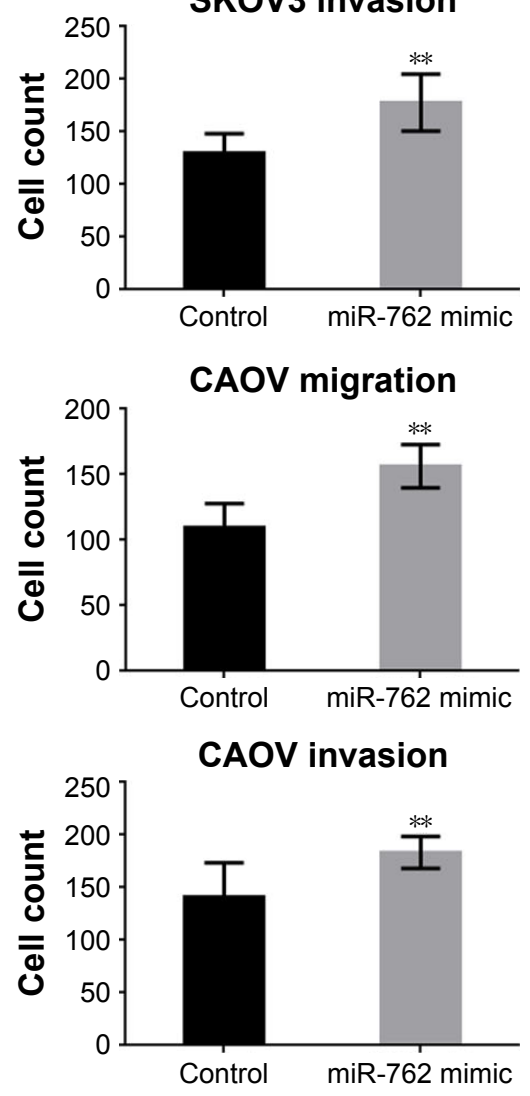

D

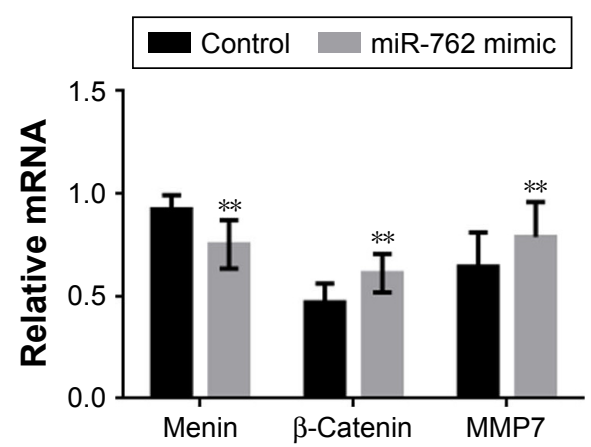

Figure 4 (Continued) 
$\mathbf{E}$

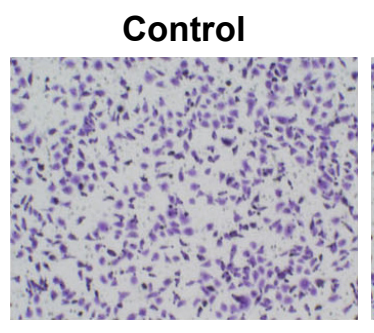

miR-762 inhibitor

$\mathbf{F}$

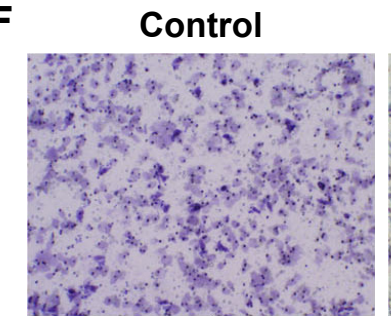

Control

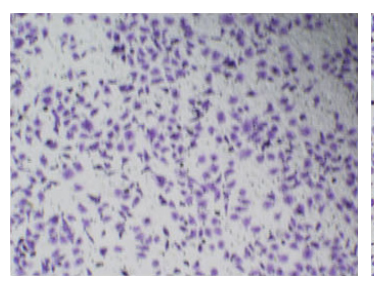

miR-762 inhibitor

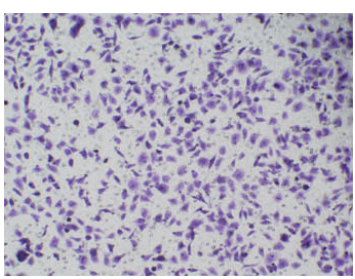

miR-762 inhibitor

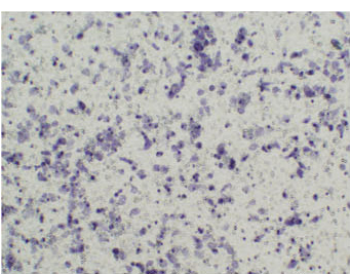

miR-762 inhibitor

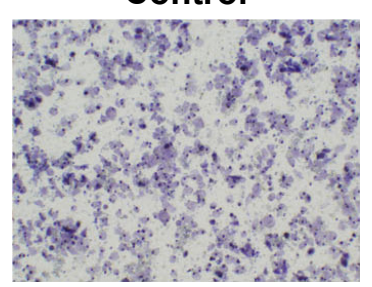

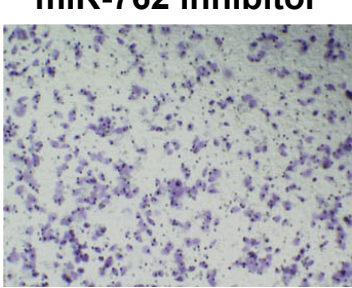

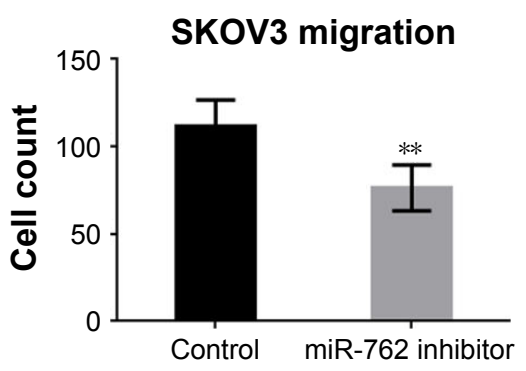

CAOV migration

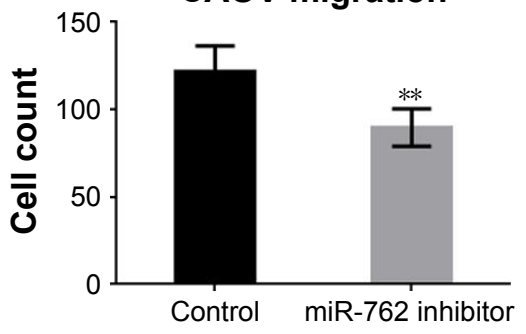

CAOV migration

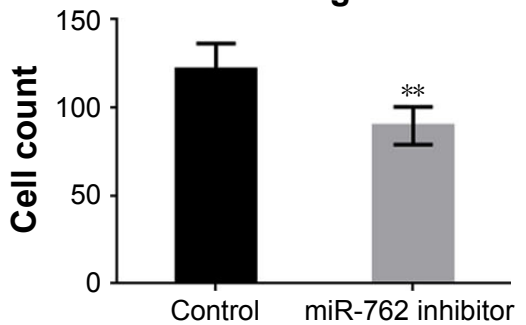

CAOV invasion

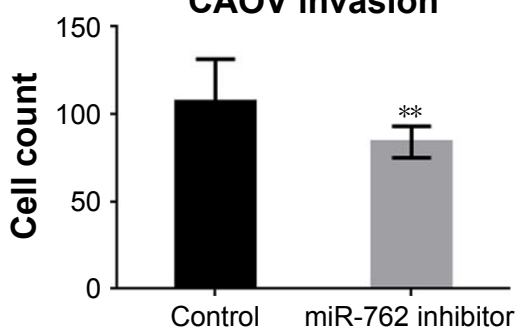

H
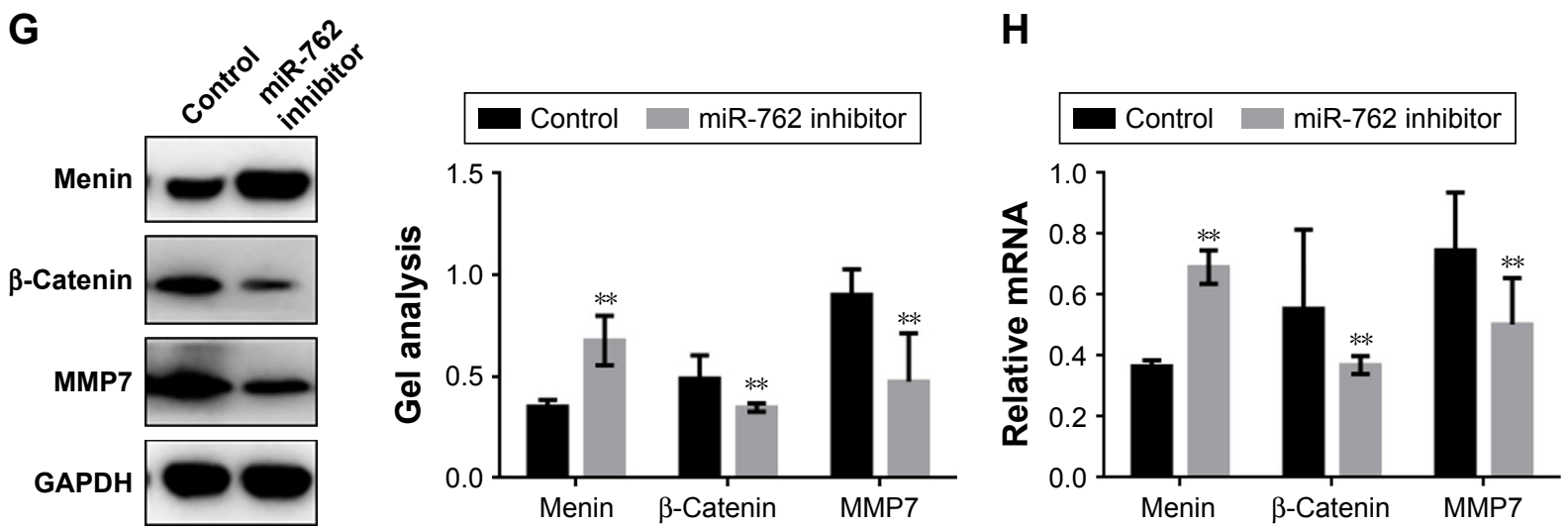

Figure 4 miR-762 promotes the metastasis of ovarian cancer cells by the suppression of menin.

Notes: (A and B) After overexpression of miR-762 in SKOV3 and CAOV cells, transwell assays with or without Matrigel were performed. Cells were counted and results represent the means $\pm S D$ of three experiments. ${ }^{* * P}<0.01$. ( $C$ and $\left.\mathbf{D}\right)$ After transfecting miR-762 mimic into SKOV3 cells, the protein and mRNA levels of menin, $\beta$-catenin, and MMP7 were detected by Western blotting and real-time PCR. Data are presented as mean \pm SEM. $* * P<0.01$. (E and $\mathbf{F}$ ) After downregulation of miR-762 in SKOV3 and CAOV cells, transwell assays with or without Matrigel were performed. Cells were counted, and the results represent the mean $\pm S D$ of three experiments. $* * P<0.0$ I. $(\mathbf{G}$ and $\mathbf{H})$ After transfection of miR-762 inhibitor into SKOV3 cells, the protein and mRNA levels of menin, $\beta$-catenin, and MMP7 were detected by Western blotting and real-time PCR. Data are presented as mean \pm SEM. $* * P<0.01$.

Abbreviations: SEM, standard error of mean; PCR, polymerase chain reaction; GAPDH, glyceraldehyde-3-phosphate dehydrogenase. 
analysis showed that miR-762 expression exhibited a negative correlation with that of menin $(y=-0.9604 x+1.211$; $R^{2}=0.7184$ ). Furthermore, miR-762 was predicted to have a binding site in the $3^{\prime}$-UTR of menin by bioinformatic analysis and reporter gene experiments confirmed that miR-762 could directly inhibit menin expression. When miR-762 was overexpressed or downregulated in ovarian cancer cells, their proliferation was promoted or suppressed, respectively. Since miR-762 could potentially promote the proliferation of ovarian cancer cells through regulation of the expression of menin, we investigated whether miR-762 could also function in ovarian cancer cell apoptosis. BAX and BCL-2 are important downstream proteins in the $\mathrm{Wnt} / \beta$-catenin signaling pathway, which regulate apoptosis. Our results also demonstrated an effect of miR-762 on apoptosis in SKOV3 cells. The regulatory effect of miR-762 on cell proliferation was achieved through its inhibition of menin and a consequent influence on the Wnt/ $\beta$-catenin signal pathway. $\beta$-Catenin is the most critical molecule in the Wnt signaling pathway and is responsible for its activation. $\beta$-Catenin can influence metastasis in numerous ways, including via its actions on epithelial-mesenchymal transition and via MMPs, specifically the common $\beta$-catenin/MMP7 pathway. We hypothesized that miR-762 could promote ovarian cancer cell metastasis through promotion of the $\beta$-catenin/MMP7 pathway. The results of transwell assays demonstrated that miR-762 could promote the invasion and migration of both SKOV3 and CAOV cells through regulation of the expression of menin, $\beta$-catenin, and MMP7.

\section{Conclusion}

In this study, we confirmed that there is a specific relationship between miR-762 and the occurrence and development of ovarian cancer. MiR-762 can directly target menin to regulate the $\mathrm{Wnt} / \beta$-catenin signaling pathway through the inhibition of menin expression. MiR-762 can also promote the proliferation and metastasis of ovarian cancer cells through negative regulation of menin expression. Our findings suggest that miR-762 may be a potential therapeutic target in ovarian cancer.

\section{Disclosure}

The authors report no conflicts of interest in this work.

\section{References}

1. Chen Y, Wang X, Duan C, et al. Loss of TAB3 expression by shRNA exhibits suppressive bioactivity and increased chemical sensitivity of ovarian cancer cell lines via the NF-kappaB pathway. Cell Prolif. 2016;49(6):657-668.
2. Latha TS, Panati K, Gowd DS, Reddy MC, Lomada D. Ovarian cancer biology and immunotherapy. Int Rev Immunol. 2014;33(5):428-440.

3. Nawaz M, Fatima F, Nazarenko I, et al. Extracellular vesicles in ovarian cancer: applications to tumor biology, immunotherapy and biomarker discovery. Expert Rev Proteomics. 2016;13(4):395-409.

4. Brouwer J, Kluiver J, de Almeida RC, et al. Small RNA sequencing reveals a comprehensive miRNA signature of BRCA1-associated highgrade serous ovarian cancer. J Clin Pathol. Epub 2016 Apr 5.

5. Erhard F, Haas J, Lieber D, et al. Widespread context dependency of microRNA-mediated regulation. Genome Res. 2014;24(6):906-919.

6. Suzuki HI, Mihira H, Watabe T, Sugimoto K, Miyazono K. Widespread inference of weighted microRNA-mediated gene regulation in cancer transcriptome analysis. Nucleic Acids Res. 2013;41(5):e62.

7. Krol J, Loedige I, Filipowicz W. The widespread regulation of microRNA biogenesis, function and decay. Nat Rev Genet. 2010;11(9):597-610.

8. Iorio MV, Visone R, Di Leva G, et al. MicroRNA signatures in human ovarian cancer. Cancer Res. 2007;67(18):8699-8707.

9. Calura E, Fruscio R, Paracchini L, et al. MiRNA landscape in stage I epithelial ovarian cancer defines the histotype specificities. Clin Cancer Res. 2013;19(15):4114-4123.

10. Vang $\mathrm{S}$, Wu HT, Fischer A, et al. Identification of ovarian cancer metastatic miRNAs. PLoS One. 2013;8(3):e58226.

11. Gao F, Chen S, Sun M, et al. MiR-467a is upregulated in radiationinduced mouse thymic lymphomas and regulates apoptosis by targeting Fas and Bax. Int J Biol Sci. 2015;11(1):109-121.

12. Li Y, Huang R, Wang L, et al. microRNA-762 promotes breast cancer cell proliferation and invasion by targeting IRF7 expression. Cell Prolif. 2015;48(6):643-649.

13. Yu T, Wang XY, Gong RG, et al. The expression profile of microRNAs in a model of 7,12-dimethyl-benz[a]anthrance-induced oral carcinogenesis in Syrian hamster. J Exp Clin Cancer Res. 2009; 28:64.

14. Papi L, Palli D, Masi L, et al. Germline mutations in MEN1 and BRCA1 genes in a woman with familial multiple endocrine neoplasia type 1 and inherited breast-ovarian cancer syndromes: a case report. Cancer Genet Cytogenet. 2009;195(1):75-79.

15. Pfaffl MW. A new mathematical model for relative quantification in real-time RT-PCR. Nucleic Acids Res. 2001;29(9):e45.

16. Yang TS, Yang XH, Wang XD, Wang YL, Zhou B, Song ZS. MiR-214 regulate gastric cancer cell proliferation, migration and invasion by targeting PTEN. Cancer Cell Int. 2013;13(1):68.

17. Hashimoto M, Kyo S, Hua X, et al. Role of menin in the regulation of telomerase activity in normal and cancer cells. Int J Oncol. 2008;33(2):333-340.

18. Imachi H, Murao K, Dobashi H, et al. Menin, a product of the MENI gene, binds to estrogen receptor to enhance its activity in breast cancer cells: possibility of a novel predictive factor for tamoxifen resistance. Breast Cancer Res Treat. 2010;122(2):395-407.

19. Imachi H, Yu X, Nishiuchi T, Miyai Y, Masugata H, Murao K. Raloxifene inhibits menin-dependent estrogen receptor activation in breast cancer cells. J Endocrinol Invest. 2011;34(11):813-815.

20. Ren F, Xu HW, Hu Y, et al. Expression and subcellular localization of menin in human cancer cells. Exp Ther Med. 2012;3(6):1087-1091.

21. Yokoyama A, Cleary ML. Menin critically links MLL proteins with LEDGF on cancer-associated target genes. Cancer Cell. 2008; 14(1):36-46.

22. Wu T, Hua X. Menin represses tumorigenesis via repressing cell proliferation. Am J Cancer Res. 2011;1(6):726-739.

23. Cao Y, Liu R, Jiang X, et al. Nuclear-cytoplasmic shuttling of menin regulates nuclear translocation of $\{$ beta $\}$-catenin. Mol Cell Biol. 2009;29(20):5477-5487. 


\section{Publish your work in this journal}

OncoTargets and Therapy is an international, peer-reviewed, open access journal focusing on the pathological basis of all cancers, potential targets for therapy and treatment protocols employed to improve the management of cancer patients. The journal also focuses on the impact of management programs and new therapeutic agents and protocols on
Dovepress

patient perspectives such as quality of life, adherence and satisfaction. The manuscript management system is completely online and includes a very quick and fair peer-review system, which is all easy to use. Visit http://www.dovepress.com/testimonials.php to read real quotes from published authors.

\footnotetext{
Submit your manuscript here: http://www.dovepress.com/oncotargets-and-therapy-journal
} 